\title{
Stem cell therapy for treating osteonecrosis of the femoral head: From clinical applications to related basic research
}

\author{
Rui Li ${ }^{1}$, Qiu-Xia Lin ${ }^{1}$, Xue-Zhen Liang ${ }^{2}$, Guang-Bo Liu' ${ }^{1}$, He Tang ${ }^{1}$, Yu Wang ${ }^{1}$, Shi-Bi Lu ${ }^{1 *}$ and Jiang Peng ${ }^{*}$
}

\begin{abstract}
Osteonecrosis of the femoral head (ONFH) is a refractory disease that is associated with collapse of the femoral head, with a risk of hip arthroplasty in younger populations. Thus, there has been an increased focus on early interventions for ONFH that aim to preserve the native articulation. Stem cell therapy is a promising treatment, and an increasing number of recent studies have focused on this topic. Many clinical studies have reported positive outcomes of stem cell therapy for the treatment of ONFH. To improve the therapeutic effects of this approach, many related basic research studies have also been performed. However, some issues must be further explored, such as the appropriate patient selection procedure, the optimal stem cell selection protocol, the ideal injection number, and the safety of stem cell therapy. The purpose of this review is to summarize the available clinical studies and basic research related to stem cell therapy for ONFH.
\end{abstract}

Keywords: Hip, Osteonecrosis, Cell therapy, Mesenchymal stem cells

\section{Background}

Osteonecrosis of the femoral head (ONFH) is a refractory disease that is characterized by compromised subchondral microcirculation, necrosis of the bone, and microfracture accumulation without sustained remodeling $[1,2]$. ONFH is a global problem, and an estimated 20,000 to 30,000 new patients are diagnosed with osteonecrosis annually in the United States [3]; in addition,

\footnotetext{
* Correspondence: lushibi301@126.com; pengjiang301@126.com ${ }^{1}$ Institute of Orthopedics, Beijing Key Laboratory of Regenerative Medicine in Orthopedics, Key Laboratory of Musculoskeletal Trauma \& War Injuries, Chinese PLA General Hospital, Beijing 100853, China

Full list of author information is available at the end of the article
}

8.12 million cases of nontraumatic ONFH are diagnosed annually among the Chinese general population aged 15 years and older [4].

Although total hip arthroplasty (THA) can provide satisfactory clinical outcomes for hip dysfunction patients, one challenge for surgeons is that ONFH occurs predominantly in patients aged 30 to 40 years. The outcomes of THA for these young and active patients are not ideal, primarily due to the limited lifetime and durability of the prosthesis. Thus, there has been an increasing focus on early interventions for ONFH that aim to preserve the native articulation. A wide variety of joint-preserving methods have been reported, including pharmacologic or physical treatment and surgical techniques ranging from core decompression $(\mathrm{CD})$ to various vascularized and nonvascularized bone-grafting procedures [5]. However, the outcomes of these studies have varied. Thus, studies that aim to identify a better treatment are ongoing.

Stem cells are a group of cells with the ability to self-renew and form differentiated cells. These cells play important roles in development and disease. They are also important "seed" cells in the process of regenerative therapy. Stem cell research is currently focused mainly on adult stem cells, embryonic stem cells and induced pluripotent stem cells. Adult stem cells, which include mesenchymal stem cells (MSCs), have been reported as a promising approach for the regeneration of various tissues. MSCs were first described in human bone marrow and called bone marrow stem cells (BMSCs); these cells can be isolated from many other sources, including adipose tissue, the synovial membrane and the umbilical cord, in addition to the bone marrow [6, 7]. Since the injection of autologous MSCs combined with standard CD for treating ONFH was first described in 1993 and the first mid-term results were reported in 2002 [8], there has been an increased focus on this approach [9]; with the development of both the technology and the 
concept, stem cell therapy has been shown to be a promising approach for treating ONFH.

The aim of this paper is to present a review of current clinical and basic research related to stem cell therapy for treating $\mathrm{ONFH}$.

\section{Outcomes in clinical applications \\ General outcome}

An increasing number of clinical studies have evaluated the therapeutic effect of stem cells on ONFH in recent years. Studies with high levels of evidence (Levels I and II) are shown in Table 1. Most of the authors demonstrated positive clinic outcomes, including reduced pain, improved function and motion, delayed progression or the avoidance of THA [10-17]. However, several researchers had reservations about this approach. Pepke et al. reported that there was no significant benefit from the additional injection of concentrated bone marrow aspirate compared with the effects of CD alone in the short term (Level of Evidence: I) [18]. In addition, several retrospective comparative studies (Level of Evidence: III) drew conclusions similar to those reported by Pepke [19, 20]. A recent meta-analysis showed that the implantation of autologous MSCs into the CD track, particularly in the early (precollapse) stages of $\mathrm{ONFH}$, could improve the survivorship of femoral heads and reduce the need for hip arthroplasty [21]. Another meta-analysis (including eight randomized controlled trials) also demonstrated that compared with $\mathrm{CD}$ alone, the combination of $\mathrm{CD}$ with regenerative techniques provides a significant improvement in survivorship over time [9].

Thus, although some controversy exists, it seems that the general outcomes of the use of stem cells to treat ONFH are positive. The reasons for the different conclusions may be the heterogeneity among studies, including differences in patient selection, cell harvesting, cell processing, and cell delivery. Thus, these heterogeneities warrant further investigation.

\section{Patient selection}

Numerous studies confirmed that the outcome of treatment was ascociated with patient condition. The most important factor may be the stage of ONFH. Ma et al. reported that the stage of ONFH might affect the outcome of stem cell therapy [14]. Hauzeur et al. reported that the implantation of bone marrow aspirate concentrate (BMAC) after $\mathrm{CD}$ did not produce any improvement in the evolution of stage III ONFH [22]. Thus, stage III and stage IV cases may be prone to poor outcomes, and early-stage (stage I or II) patients should be a more appropriate choice. In addition, Sen et al. [12] reported that patients with posttraumatic osteonecrotic hips had better outcomes than did patients with nontraumatic hips, which suggested that etiology is another a factor that affects clinical outcomes. Furthermore,
Houdek et al. suggested that patients with a low modified Kerboul grade may achieve better results [23]. It seems that the stage, size, morphology and even etiology of ONFH may be important factors associated with the treatment outcome. Thus, to achieve better results, it is critical to select appropriate cases.

Moreover, it has been reported that aging is associated with decreases in the number of MSCs isolated from a donor and the proliferation ability of those cells [24, 25]. Stenderup et al. [26] found that although MSC function was decreased in cells isolated from older donors in vitro, this difference did not affect the ability of the cells to differentiate in vivo. The authors concluded that MSCs isolated from older donors maintained normal cellular function but showed a proliferative defect. In addition, Aksu et al. [27] found that sex may affect the differentiation potential of human adipose-derived stem cells. However, Sen et al. [12] reported that patient variables, such as sex differences, side of involvement, and opposite side involvement, had no effect on outcomes. Whether these factors influence the treatment efficiency in ONFH patients has not been well studied. Additional studies that are focused on subgroup analysis and the proper inclusion criteria for stem cell therapy in ONFH patients are needed in the future.

\section{Cell selection}

Various types of MSCs have been used to treat ONFH, including bone marrow-derived MSCs (BMMSCs), adipose-derived MSCs (ADMSCs), allogeneic human umbilical cord-derived MSCs (hUCMSCs) and peripheral blood MSCs (PBMSCs). Among the various kinds of MSCs derived from different tissues, BMMSCs are the most commonly used type. BMMSCs are used mostly as bone marrow concentrate (BMC) and are more rarely cultured or used simply as bone marrow aspirates [9]. Rastogi et al. [13] compared isolated mononuclear cells with unprocessed bone marrow injections and found that there were considerable improvements in hip function, as measured by the Harris hip score, in both groups. There was a decrease in the lesion size in the processed isolated mononuclear cell group, and 3 of 30 hips in the unprocessed bone marrow injection group required total hip replacement. It seems that the more effective procedure had better outcomes than did unprocessed bone marrow injection for the treatment of ONFH.

In addition to BMMSCs, ADMSCs are another choice for cytotherapy in patients with ONFH. This method of acquiring MSCs is not only less expensive but also less invasive and painful than that used for bone marrow harvesting [28]. An in vitro study demonstrated that adipose-derived MSCs may provide a more robust growth rate and bone differentiation potential than bone 


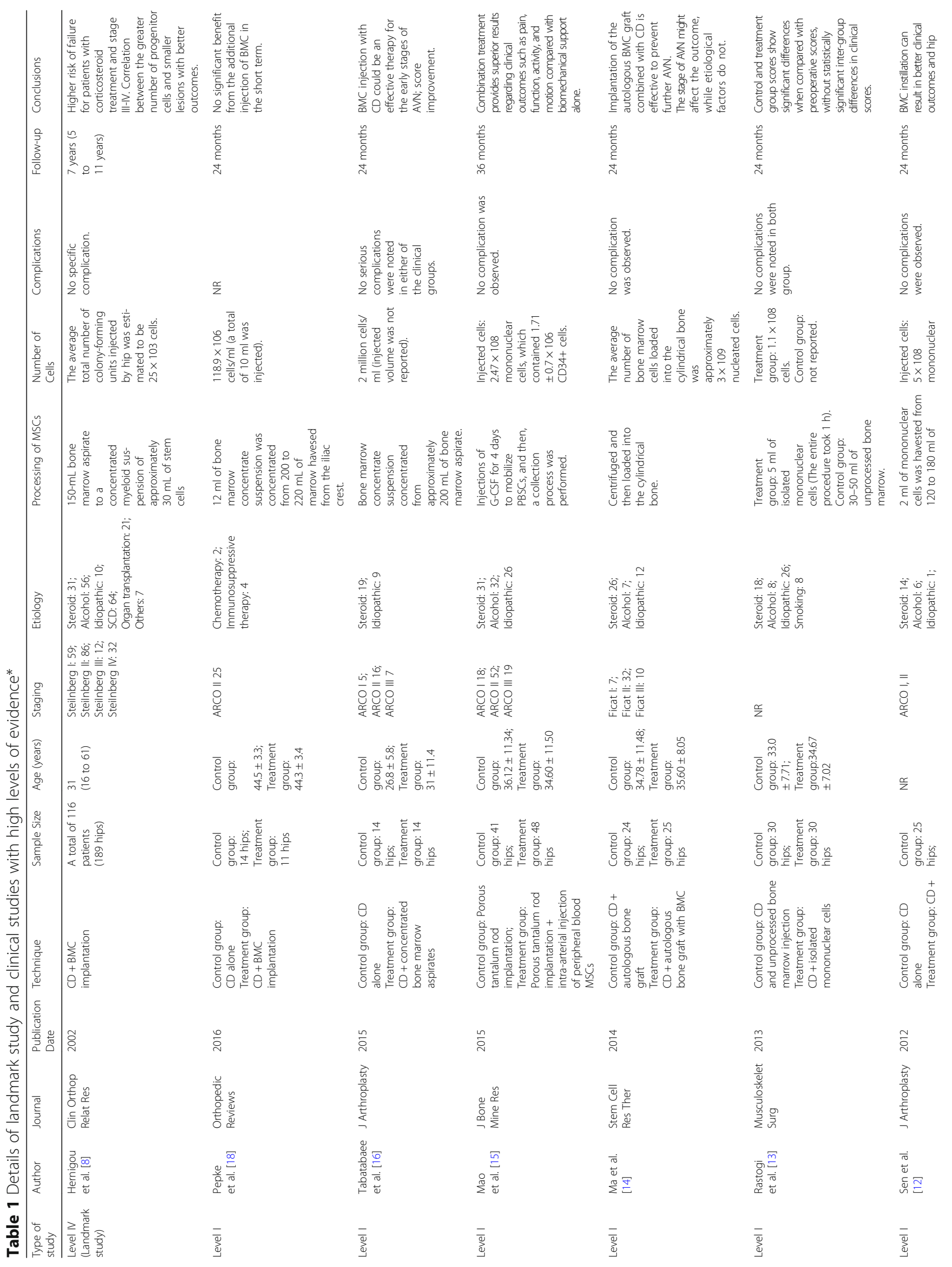




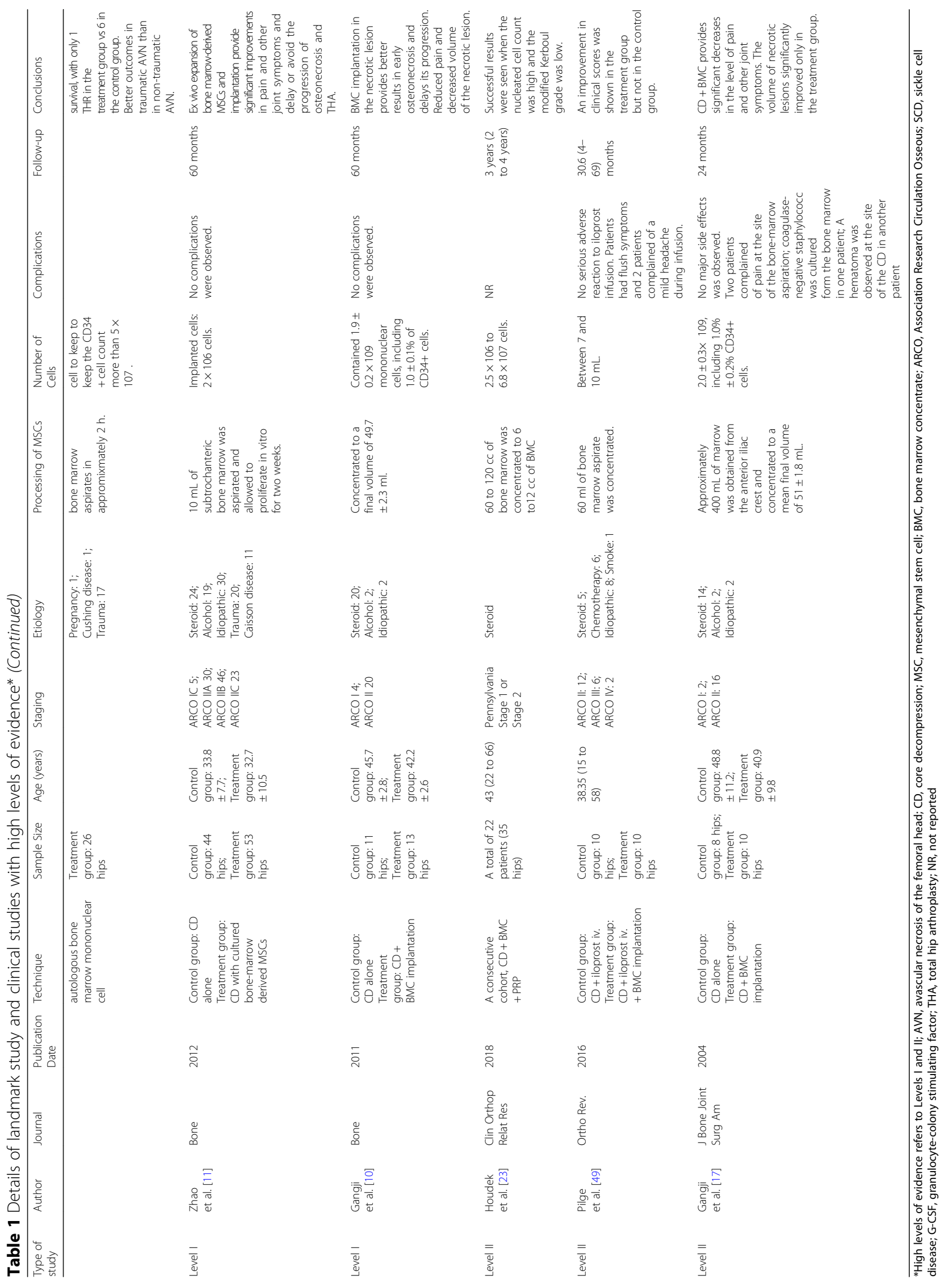


marrow-derived MSCs [29]. As adipose-derived MSCs are more abundant and show a superior functional phenotype for this purpose, they may prove to be a more effective therapeutic approach. Although the results of these studies were promising, there is a lack of well-designed prospective in vivo clinical studies to further confirm this conclusion.

Moreover, it has been demonstrated that the osteogenesis and proliferation of MSCs are decreased in alcohol-induced and steroid-induced ONFH patients [30-33]. Therefore, the transfusion of autologous stem cells isolated from these patients may have different therapeutic effects. Thus, allogeneic stem cells derived from healthy humans may be an alternative for treating ONFH. Interestingly, there is evidence for the accumulation of low-immunogenicity MSCs, which allows the MSCs to be transplanted between human leukocyte antigen (HLA)-incompatible individuals [34]. hUCMSCs may be a good candidate for this approach, because umbilical cord (UC) collection is easy and ethically feasible. The yield of UCMSCs is high, and the cells have low immunogenicity. UCMSCs are easy to separate and can be amplified in vitro; placental UCMSCs can typically be passaged for 30-40 generations, while adult BMMSCs can grow only 6-10 generations with the same performance.

Cai et al. [35] evaluated the cotransplantation of autologous BMMSCs and allogeneic UCMSCs for treating ONFH and observed therapeutic effects without severe adverse effects at 12 months after transplantation. Chen et al. [36] analyzed the clinical effects of transplanting allogeneic hUCMSCs for the treatment of ONFH and achieved clear results with no obvious side-effects after a three-year follow-up. However, there were only 30 cases and 9 cases in the studies of Cai et al. and Chen et al., respectively. Studies with larger numbers of patients and longer follow-up times are needed to further evaluate the efficiency and safety of the use of allogeneic hUCMSCs in treating ONFH.

\section{Number of injected cells}

The prevalence of connective tissue progenitors in the bone marrow in the iliac crests of patients was approximately one per 30,000 nucleated cells [37]. Hernigou et al. reported that according to the mean nucleated cell count per $\mathrm{ml}\left(18 \times 10^{6}\right.$ cells $)$, the bone marrow harvested from the iliac crest by aspiration contained an average of approximately 600 progenitors per $\mathrm{ml}$ [38]. If expansion is performed in vitro, more cells will be harvested.

It was reported that good outcomes may be associated with high nucleated cell counts [20, 23]. However, the optimum number of cells for injection remains unknown. The average volume repair was $15 \mathrm{~cm}^{3}$ in a series of osteonecrosis patients, as indicated by MRI observations and histologic observations that demonstrated that the proportion of trabecular bone was $1 / 3$ in the femoral head, with the other $2 / 3$ being fat and hematologic cells [39]. Based on a mean bone matrix of $33 \%$ in cancellous bone, it was estimated that there are approximately 20 million osteoblasts or osteocytes per $\mathrm{cm}^{3}$ of new bone [39]. Thus, approximately $3 \times 10^{8}$ (20 million cells $/ \mathrm{cm}^{3} \times 15 \mathrm{~cm}^{3}$ ) osteoblasts or osteocytes are needed for new bone repair. However, achieving an objective number of osteoblasts or osteocytes depends not only on the number of stem cells injected but also on how many times the stem cells can proliferate and how many cells can effectively differentiate into osteoblasts or osteocytes, especially in the ischemic and anoxic microenvironment of the necrotic area of the femoral head. On the other hand, whether the injection of more stem cells is better and whether there is a safe threshold for the maximum injection of stem cells remain unknown.

Based on current reported studies, except for patients injected with approximately $24 \times 10^{3}$ to $25 \times 10^{3}$ cells in early studies reported by Hernigou et al. [8, 40], the number of cell used in most other studies ranged from $10^{6}$ to $10^{9}$, and the most frequently used number was $10^{8}$ cells $[11-13,18,20]$. Thus, based on current data, the injection of $10^{6}$ to $10^{9}$ cells may be reasonable. However, the optimal number still needs to be investigated.

\section{Delivery techniques and combined treatment}

Various techniques for cell delivery have been reported in recent studies, and such techniques were commonly combined with CD $[10-12,16,18]$. Other techniques included impaction allogeneic bone grafting $[41,42]$, autoiliac cancellous bone grafts $[43,44]$, porous tantalum rod implantation procedures [15], porous tantalum rod implantation combined with vascularized iliac grafting [45], interconnected porous calcium hydroxyapatite (IP-CHA) [46] and porous nanohydroxylapatite [47].

In addition to the topical application of MSCs in the necrotic zone of the femoral head, some studies also applied the MSCs through arterial injection. Cai et al. transplanted MSCs into the medial circumflex femoral artery, the lateral circumflex femoral artery or the obturator artery through digital subtraction angiography and observed a therapeutic effect on avascular necrosis of the femoral head (ANFH) without severe adverse effects [35]. Mao et al. reported the intra-arterial infusion of PBMSCs and found that this approach could enhance the efficacy of biomechanical support during the treatment of ONFH [15]. These two studies demonstrated that intra-artery infusion could be another effective way to treat ONFH. In addition, these studies also provided evidence that MSCs could effectively act on ischemic areas. However, determining whether the topical application or intra-arterial infusion of MSCs is more effective requires further investigation. 
Some studies also combined local injection with platelet-rich plasma (PRP) [48], pharmacological treatments, such as intravenous iloprost [49] and oral bisphosphonates [19], or physical therapy, such as low-intensity pulsed ultrasound (LIPUS) [50]. Most of these studies reported satisfactory outcomes, but some studies had lower levels of evidence; thus, whether such combinations support better outcomes must be further confirmed. Moreover, comparisons between various methods have rarely been reported.

Note that, in general, regardless of which delivery technique and combined treatment were used, all of the approaches yielded improved results.

\section{Safety}

One of the major concerns in cell therapy is safety. Stem cells have some features of cancer cells, including a long lifespan, relative apoptosis resistance, and the ability to replicate for extended periods of time. In addition, similar growth regulators and control mechanisms are involved in both cancer and stem cell maintenance. Therefore, stem cells may undergo malignant transformation, which is often seen as a key obstacle to the safe use of stem-cell-based medicinal products [51]. It was reported that the transplantation of embryonic stem cells may increase the risk of teratoma formation [52]. Other concerns, including immune rejection and genetic modification, also limit the clinical use of directly transplanted stem cells for ONFH.

After a review of current studies that used stem cells in the treatment of ONFH, we found that most studies reported that no severe complications were observed. Only a few studies reported that patients had complications, such as flushing, mild headache and fever [44, 49]. Thus, based on the current studies, it seems that the application of stem cells for the treatment of ONFH is relatively safe. However, additional studies and long-term follow-up are still needed to further confirm this conclusion.

In addition, for cell therapy, which requires cell expansion in vitro, the entire process must be supervised to ensure that the cells maintain their overall phenotype and functional potential and to ensure that the cultured cells remain untransformed with no microbiological contamination [51]. Thus, standardization with respect to the quantitative and qualitative characterization of cellular therapies may need to be established in the future.

\section{Advances in related basic research Rationale behind cell therapy}

Although overall, clinical trials have achieved promising results, it is undeniable that a few studies were not valid. Thus, stem cell therapy remains controversial, which has limited its widespread use. Therefore, to improve the treatment effect of stem cell therapy, many exploratory studies, including cell experiments and animal experiments, have been performed.

A major concern of stem cell therapy is the fate and potential osteogenic activity of MSCs in the ischemic and hypoxic microenvironment at the osteonecrotic site, where the apoptosis of bone cells may occur [53]. It was reported that transplanted stem cells exhibit a low survival rate in ischemic tissue [54].

Yan et al. [55] transplanted autologous MSCs after decompression in traumatic dog models. They found that green fluorescent protein (GFP)-labeled MSCs were present in the necrotic area up to 12 weeks after transplantation, and their number increased from $15 \%$ in the 2 nd week to $38 \%$ in the 12th week. Immunohistochemical staining for osteocalcin was positive in $90 \%$ of the GFP-labeled MSCs in vivo. The percentages of trabecular bone volume were $9.36 \%$ and $8.42 \%$ in the 2 nd week ( $p>0.05), 22.82 \%$ and $14.72 \%$ in the 8 th week, and $31.08 \%$ and $20.66 \%$ in the 12 th week for the MSC-treated and control groups, respectively, and new trabecular bone in the MSC-transplanted group was significantly increased compared to that of the saline (control) group in the 8th and 12th weeks. This finding demonstrated that the transplanted MSCs could survive, proliferate, and differentiate into osteoblasts directly.

Jin et al. [56] drew a conclusion similar to that reported by Yan. They found that intra-arterially infused MSCs could migrate into the necrotic field in the femoral head and differentiate into osteoblasts, improving necrosis of the femoral head.

Moreover, Ciapetti et al. demonstrated that proliferation, colony formation and osteogenic commitment are not hampered by a low- $\mathrm{O}_{2}$ microenvironment [57]. In their study, the cells were expanded and induced to undergo osteogenic differentiation under a $2 \% \mathrm{pO}_{2}$ atmosphere (hypoxia), in contrast to their behavior under the standard $21 \% \mathrm{pO}_{2}$ atmosphere (normoxia). Those authors found that both proliferation and colony-forming ability were significantly enhanced in hypoxia-exposed BMMSCs compared with those of BMMSCs grown under normoxic conditions. The expression of bone-related genes, including alkaline phosphatase, type I collagen, and osteocalcin, was significantly increased under hypoxic conditions. Moreover, mineral deposition after osteogenic induction was not hampered and was even enhanced in some cases under low oxygen tension. These findings suggest that MSCs can survive and maintain their function in a hypoxic microenvironment.

Fan et al. [58] found that compared with BMMSCs from normal rabbits, BMMSCs from osteonecrotic rabbits showed a significantly reduced proliferative ability, reduced expression of stemness genes, decreased osteoblast formation, and increased adipocyte formation. However, after low-oxygen (2\%) treatment, BMMSCs from osteonecrotic rabbits showed not only increased 
proliferation and osteogenic potential but also decreased adipogenic potential. Further, those authors demonstrated that the transplantation of $2 \% \mathrm{O}_{2}$ versus $20 \% \mathrm{O}_{2}$ MSCs after $\mathrm{CD}$ resulted in an increase in angiogenic function and a decrease in local tissue apoptosis in a rabbit model. Hypoxia-preconditioned BMMSCs could reverse the impairment of osteonecrotic BMMSCs and enhance their therapeutic effects [59]. Other studies drew the similar conclusion that an osteogenic phenotype can be promoted if MSCs are exposed to hypoxia during the initial steps of differentiation [60-62].

Thus, transplanted cells can survive, proliferate, and differentiate into osteoblasts in osteonecrotic areas in animal models. Interestingly, a low- $\mathrm{O}_{2}$ microenvironment does not seem to harm MSCs and even promoted the osteogenic phenotype in cellular experiments; these findings must be confirmed in humans.

\section{Subpopulations of MSCs}

A definition for MSCs was provided by the International Society for Cellular Therapy in 2006 [63]. First, MSCs must be plastic adherent when maintained under standard culture conditions. Second, MSCs must express CD105, CD73 and CD90 and lack the expression of the CD45, CD34, CD14 or CD11b, CD79alpha or CD19 and HLA-DR surface molecules. Third, MSCs must differentiate into osteoblasts, adipocytes and chondroblasts in vitro. Although this definition is currently widely accepted, evidence that MSC subpopulations may feature distinct characteristics and regeneration potentials has been reported, and different subpopulations have distinct potentials to promote differentiation into osteoblasts or chondrocytes [64].

Levi et al. [65] demonstrated that compared with either CD105(high) or unsorted cells, FACS-sorted CD105(low) ADMSCs were significantly enhanced in osteogenic differentiation and bone regeneration; this effect is likely due to reduced TGF-beta1/Smad2 signaling. Additionally, Leyva-Leyva et al. [66] observed that CD105- cells showed stronger expression of secreted protein acidic and rich in cysteine (SPARC) and osteonectin, which was associated with more effective calcium deposition, than did CD105+ cells. Furthermore, through in vivo trials, it was demonstrated that grafts containing CD105- cells promoted adequate graft integration, improved host vascular infiltration, and facilitated efficient repair through intramembranous ossification. By contrast, grafts containing CD105+ cells showed abundant fibrocartilaginous tissue and deficient endochondral ossification [67].

While conflicting data have been reported for BMSCs, Aslan et al. [68] found that in the bone marrow, CD105+ cells displayed significantly more fibroblast colony-forming units than did unseparated mononuclear cells. Similarly, Jarocha et al. [69] reported that expanded CD105 and
CD271 populations possess higher expression levels of RUNX2 and OCN.

CD90 is another marker for subpopulation selection. Yamamoto [70] found that CD90+ cells are more capable of forming bone both in vitro and in vivo. In addition, CD90 may be a more effective marker than CD105 for isolating a highly osteogenic subpopulation. Rada et al. [71] reported that the cells isolated with an anti-CD90 antibody (ab), an anti-CD49d ab and an anti-p75 ab exhibited a high osteogenic differentiation potential but demonstrated the lowest chondrogenic differentiation potential. On the other hand, the cells isolated with an anti-CD73 ab exhibited a high chondrogenic differentiation potential but the lowest osteogenic potential.

In addition, Harumichi et al. [72] reported that although significant differences in proliferation capacity were not seen, the adipogenic and osteogenic differentiation capacities were higher in aldehyde dehydrogenase (ALDH)-high subpopulations than in ALDH-low subpopulations. All these studies revealed that osteogenic potential is related to different subpopulations.

To date, preclinical and clinical studies of ONFH treatments used unsorted MSCs, which may consist of various cell subpopulations; this factor may be one of the most important reasons for the inconsistent results of previous studies. Thus, the accurate selection of a subpopulation may enhance treatment efficiency for $\mathrm{ONFH}$ and may be a direction for future research.

\section{Gene-modified MSCs}

Some studies have also focused on the use of genetic engineering to modify MSCs, such as bone morphogenetic protein-2 (BMP-2), basic fibroblast growth factor (bFGF), hepatocyte growth factor (HGF), vascular endothelial growth factor (VEGF), calcitonin generelated peptide (CGRP) or their combinations.

Tang et al. [73] used BMP-2-modified BMMSCs to repair experimentally induced $\mathrm{ONFH}$ in goats and obtained good results. Pend et al. demonstrated that BMP-2- and bFGFmodified BMSCs could successfully repair ONFH in a dog model by promoting bone formation and angiogenesis [74]. VEGF alone or combined with BMP-2-modified MSCs also yielded positive results in dog and rabbit experiments $[75,76]$.

In addition, the ability of HGF, a pleiotropic cytokine, to exert potent mitogenic effects and promote nutrient absorption and utilization to facilitate tissue repair in the liver, heart and muscle was also evaluated. Wen et al. [77] demonstrated that the combination of $\mathrm{CD}$ and the transplantation of HGF-transgenic autologous BMSCs enhanced blood vessel regeneration and bone reconstruction in a rabbit ANFH model. This positive outcome was also confirmed by Pan et al. [78]. 
Gene-modified MSCs may be a promising technique for improving treatment efficiency in ONFH patients. However, all these results were obtained in animal experiments. The efficiency and safety of these approaches in $\mathrm{ONFH}$ patients require further evaluation.

\section{MSC-derived exosomes for cell-free therapy}

It was originally thought that MSCs exert their therapeutic effect by migrating to sites of damage, engrafting, and subsequently differentiating into the desired cells for tissue regeneration. However, accumulated evidence has indicated that the therapeutic benefit of MSCs is attributable not only to their differentiation but also to the factors they secrete [79-81]. In addition to growth factors, cytokines, chemokines, and bioactive lipids secreted by stem cell therapeutics, cells can communicate with neighboring cells or with distant cells through the secretion of extracellular vesicles (EVs). EVs are composed of a lipid bilayer that contains transmembrane proteins and encloses cytosolic proteins and RNA. Cells can secrete different types of EVs, such as exosomes and microvesicles (MV), which have been classified according to their subcellular origins [82]. Exosomes are vesicles that are smaller than $150 \mathrm{~nm}$ in diameter and enriched in endosome-derived components [83].

Guo et al. demonstrated that exosomes from human synovial-derived MSCs could prevent glucocorticoidinduced ONFH in rats by enhancing proliferation and antiapoptotic effects [84]. Liu et al. further reported that exosomes secreted from human-induced pluripotent stem cell-derived MSCs could prevent ONFH by promoting angiogenesis [85].

This cell-free treatment plays an increasingly important role in regenerative therapy. However, unlike pharmaceutical treatments that deliver a single agent at a specific dose, MSCs are site-regulated and secrete bioactive factors and signals at variable concentrations in response to local microenvironmental cues [86]. Thus, whether MSC-derived vesicles can fully replace MSCs must be further evaluated.

In addition, the potential risks of using exosomes should be considered. Accumulating evidence has indicated that cells communicate via the release and delivery of microRNAs (miRNAs) packed into tumor-released (TR) exosomes [87]. It was also reported that exosome vehicles could transfer toxic proteins associated with neurodegenerative diseases [88]. Thus, the safety of using MSC-derived vesicles must also be evaluated.

Finally, there is much work to be done before MSC-derived vesicle therapy can be used clinically, including standardized production, vesicle characterization, improved isolation and yield optimization, reproducibility, the development of an assay for potency, and a determination of the doses for particular clinical indications.

\section{Conclusion}

Extensive research activities over the last decade have explored the potential of MSCs and have shown promising results in both animal experiments and clinical applications. Although some controversy exists, it seems that the general outcomes of the use of stem cells to treat ONFH are positive in terms of not only efficiency, but also safety.

For clinical applications, the different conclusions may be due to the heterogeneity among studies. It seems that patients in ARCO I and ARCO II stages and patients with a low modified Kerboul grade are good candidates for this technique. BMMSCs were still the most commonly used cells, while other types of stem cells, such as ADMSCs, show a more promising prospect, with a robust growth rate and bone differentiation potential, and could be considered as an alternative to BMMSCs. In addition, it was reported that the good outcomes may be associated with high nucleated cell counts. Although the most proper number of cells for injection was not determined, based on the current available data, injection of $10^{6}$ to $10^{9}$ cells may be reasonable. Further clinical applications should be aware of the appropriate patient selection procedure, the optimal stem cell selection protocol and the ideal injection number to achieve better outcomes.

For the related basic research, inspiring advanced progress has been made. Preconditioning of MSCs and accurate selection of a subpopulation may enhance treatment efficiency for ONFH. Use of genetic engineering to modify MSCs, such as BMP-2 and VEGF, also constituted good attempts to use MSCs more efficiently. Recently, cell-free treatment has played an increasingly important role in regenerative therapy and may develop as an alternative to stem cell therapy. However, much work must be done before these experimental approaches can be applied in clinical practice, in terms of not only efficiency, but also safety. Standardization with respect to the quantitative and qualitative characterization of cellular therapies is urgently needed in the future.

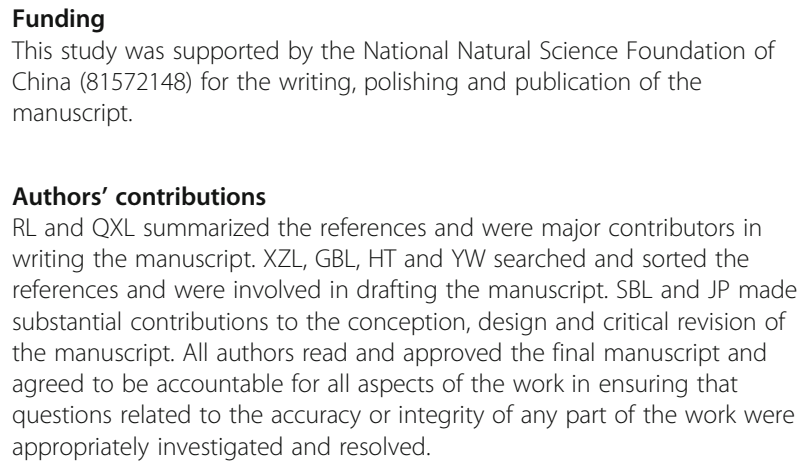

Authors' contributions

$\mathrm{RL}$ and QXL summarized the references and were major contributors in writing the manuscript. XZL, GBL, HT and YW searched and sorted the references and were involved in drafting the manuscript. SBL and JP made substantial contributions to the conception, design and critical revision of the manuscript. All authors read and approved the final manuscript and agreed to be accountable for all aspects of the work in ensuring that questions related to the accuracy or integrity of any part of the work were appropriately investigated and resolved.

Ethics approval and consent to participate Not applicable 


\section{Consent for publication}

Not applicable.

\section{Competing interests}

The authors declare that they have no competing interests.

\section{Publisher's Note}

Springer Nature remains neutral with regard to jurisdictional claims in published maps and institutional affiliations.

\section{Author details}

${ }^{1}$ Institute of Orthopedics, Beijing Key Laboratory of Regenerative Medicine in Orthopedics, Key Laboratory of Musculoskeletal Trauma \& War Injuries, Chinese PLA General Hospital, Beijing 100853, China. ${ }^{2}$ The First Clinical Medical School, Shandong University of Traditional Chinese Medicine, Jinan 250355, Shandong, China.

\section{Published online: 25 October 2018}

\section{References}

1. Moya-Angeler J, Gianakos AL, Villa JC, Ni A, Lane JM. Current concepts on osteonecrosis of the femoral head. World J Orthop. 2015;6(8):590-601 Epub 2015/09/24

2. Shah KN, Racine J, Jones LC, Aaron RK. Pathophysiology and risk factors for osteonecrosis. Curr Rev Musculoskelet Med. 2015;8(3):201-9 Epub 2015/07/06.

3. Mankin HJ. Nontraumatic necrosis of bone (osteonecrosis). N Engl J Med. 1992;326(22):1473-9 Epub 1992/05/28.

4. Zhao DW, Yu M, Hu K, Wang W, Yang L, Wang BJ, et al. Prevalence of Nontraumatic Osteonecrosis of the Femoral Head and its Associated Risk Factors in the Chinese Population: Results from a Nationally Representative Survey. Chin Med J. 2015;128(21):2843-50 Epub 2015/11/03.

5. Mont MA, Cherian JJ, Sierra RJ, Jones LC, Lieberman JR. NontraumatiC Osteonecrosis of the Femoral Head: Where Do We Stand Today? A Ten-Year Update. J Bone Joint Surg Am. 2015;97(19):1604-27 Epub 2015/10/09.

6. Friedenstein AJ, Deriglasova UF, Kulagina NN, Panasuk AF, Rudakowa SF, Luria EA, et al. Precursors for fibroblasts in different populations of hematopoietic cells as detected by the in vitro colony assay method. Exp Hematol. 1974;2(2):83-92 Epub 1974/01/01.

7. Prockop DJ. Marrow stromal cells as stem cells for nonhematopoietic tissues. Science. 1997;276(5309):71-4 Epub 1997/04/04.

8. Hernigou P, Beaujean F. Treatment of osteonecrosis with autologous bone marrow grafting. Clin Orthop Relat Res. 2002;405:14-23 Epub 2002/12/04.

9. Andriolo L, Merli G, Tobar C, Altamura SA, Kon E, Filardo G. Regenerative therapies increase survivorship of avascular necrosis of the femoral head: a systematic review and meta-analysis. Int Orthop. 2018;6 Epub 2018/02/08.

10. Gangji V, De Maertelaer V, Hauzeur JP. Autologous bone marrow cell implantation in the treatment of non-traumatic osteonecrosis of the femoral head: Five year follow-up of a prospective controlled study. Bone. 2011;49(5):1005-9 Epub 2011/08/09

11. Zhao D, Cui D, Wang B, Tian F, Guo L, Yang L, et al. Treatment of early stage osteonecrosis of the femoral head with autologous implantation of bone marrow-derived and cultured mesenchymal stem cells. Bone. 2012; 50(1):325-30 Epub 2011/11/19.

12. Sen RK, Tripathy SK, Aggarwal S, Marwaha N, Sharma RR, Khandelwal N. Early results of core decompression and autologous bone marrow mononuclear cells instillation in femoral head osteonecrosis: a randomized control study. J Arthroplast. 2012;27(5):679-86 Epub 2011/10/18.

13. Rastogi S, Sankineani SR, Nag HL, Mohanty S, Shivanand G, Marimuthu K, et al. Intralesional autologous mesenchymal stem cells in management of osteonecrosis of femur: a preliminary study. Musculoskelet Surg. 2013;97(3): 223-8 Epub 2013/07/16.

14. Ma Y, Wang T, Liao J, Gu H, Lin X, Jiang Q, et al. Efficacy of autologous bone marrow buffy coat grafting combined with core decompression in patients with avascular necrosis of femoral head: a prospective, doubleblinded, randomized, controlled study. Stem Cell Res Ther. 2014;5(5):115 Epub 2014/10/16

15. Mao Q, Wang W, Xu T, Zhang S, Xiao L, Chen D, et al. Combination treatment of biomechanical support and targeted intra-arterial infusion of peripheral blood stem cells mobilized by granulocyte-colony stimulating factor for the osteonecrosis of the femoral head: a randomized controlled clinical trial. J Bone Miner Res. 2015;30(4):647-56 Epub 2014/10/29.
16. Tabatabaee RM, Saberi S, Parvizi J, Mortazavi SM, Farzan M. Combining Concentrated Autologous Bone Marrow Stem Cells Injection With Core Decompression Improves Outcome for Patients with Early-Stage Osteonecrosis of the Femoral Head: A Comparative Study. J Arthroplast. 2015;30(9 Suppl):11-5 Epub 2015/07/06.

17. Gangji V, Hauzeur JP, Matos C, De Maertelaer V, Toungouz M, Lambermont M. Treatment of osteonecrosis of the femoral head with implantation of autologous bone-marrow cells. A pilot study. J Bone Joint Surg Am. 2004; 86-a(6):1153-60 Epub 2004/06/03.

18. Pepke W, Kasten P, Beckmann NA, Janicki P, Egermann M. Core Decompression and Autologous Bone Marrow Concentrate for Treatment of Femoral Head Osteonecrosis: A Randomized Prospective Study. Orthop Rev (Pavia). 2016;8(1):6162 Epub 2016/04/27.

19. Gianakos AL, Moya-Angeler J, Duggal S, Zambrana L, Fields KG, Mintz DN, et al. The Efficacy of Bisphosphonates with Core Decompression and Mesenchymal Stem Cells Compared with Bisphosphonates Alone in the Treatment of Osteonecrosis of the Hip: a Retrospective Study. HSS J. 2016; 12(2):137-44 Epub 2016/07/08.

20. Lim YW, Kim YS, Lee JW, Kwon SY. Stem cell implantation for osteonecrosis of the femoral head. Exp Mol Med. 2013;45:e61 Epub 2013/11/16.

21. Papakostidis C, Tosounidis TH, Jones E, Giannoudis PV. The role of "cell therapy" in osteonecrosis of the femoral head. A systematic review of the literature and meta-analysis of 7 studies. Acta Orthop. 2016;87(1):72-8 Epub 2015/07/30.

22. Hauzeur JP, De Maertelaer V, Baudoux E, Malaise M, Beguin Y, Gangji V. Inefficacy of autologous bone marrow concentrate in stage three osteonecrosis: a randomized controlled double-blind trial. Int Orthop. 2017; 7:1429-35 Epub 2017/10/11.

23. Houdek MT, Wyles CC, Collins MS, Howe BM, Terzic A, Behfar A, et al. Stem Cells Combined With Platelet-rich Plasma Effectively Treat Corticosteroidinduced Osteonecrosis of the Hip: A Prospective Study. Clin Orthop Relat Res. 2018;476(2):388-97 Epub 2018/03/13.

24. Choudhery MS, Badowski M, Muise A, Pierce J, Harris DT. Donor age negatively impacts adipose tissue-derived mesenchymal stem cell expansion and differentiation. J Transl Med. 2014;12:8 Epub 2014/01/09.

25. D'Ippolito G, Schiller PC, Ricordi C, Roos BA, Howard GA. Age-related osteogenic potential of mesenchymal stromal stem cells from human vertebral bone marrow. J Bone Miner Res. 1999;14(7):1115-22 Epub 1999/07/15.

26. Stenderup K, Justesen J, Clausen C, Kassem M. Aging is associated with decreased maximal life span and accelerated senescence of bone marrow stromal cells. Bone. 2003;33(6):919-26 Epub 2003/12/18.

27. Aksu AE, Rubin JP, Dudas JR, Marra KG. Role of gender and anatomical region on induction of osteogenic differentiation of human adipose-derived stem cells. Ann Plast Surg. 2008:60(3):306-22 Epub 2008/04/30.

28. Baer PC, Geiger H. Adipose-derived mesenchymal stromal/stem cells: tissue localization, characterization, and heterogeneity. Stem Cells Int. 2012;2012: 812693 Epub 2012/05/12.

29. Wyles CC, Houdek MT, Crespo-Diaz RJ, Norambuena GA, Stalboerger PG Terzic A, et al. Adipose-derived Mesenchymal Stem Cells Are Phenotypically Superior for Regeneration in the Setting of Osteonecrosis of the Femoral Head. Clin Orthop Relat Res. 2015;473(10):3080-90 Epub 2015/06/14.

30. Lee JS, Lee JS, Roh HL, Kim CH, Jung JS, Suh KT. Alterations in the differentiation ability of mesenchymal stem cells in patients with nontraumatic osteonecrosis of the femoral head: comparative analysis according to the risk factor. J Orthop Res. 2006;24(4):604-9 Epub 2006/03/04.

31. Suh KT, Kim SW, Roh HL, Youn MS, Jung JS. Decreased osteogenic differentiation of mesenchymal stem cells in alcohol-induced osteonecrosis. Clin Orthop Relat Res. 2005;431:220-5 Epub 2005/02/03.

32. Houdek MT, Wyles CC, Packard BD, Terzic A, Behfar A, Sierra RJ. Decreased Osteogenic Activity of Mesenchymal Stem Cells in Patients With Corticosteroid-Induced Osteonecrosis of the Femoral Head. J Arthroplast. 2016;31(4):893-8 Epub 2015/09/26

33. Wang BL, Sun W, Shi ZC, Lou JN, Zhang NF, Shi SH, et al. Decreased proliferation of mesenchymal stem cells in corticosteroid-induced osteonecrosis of femoral head. Orthopedics. 2008:31(5):444 Epub 2009/03/19.

34. Lee HJ, Kang KS, Kang SY, Kim HS, Park SJ, Lee SY, et al. Immunologic properties of differentiated and undifferentiated mesenchymal stem cells derived from umbilical cord blood. J Vet Sci. 2015;17(3):289-97.

35. Cai J, Wu Z, Huang L, Chen J, Wu C, Wang S, et al. Cotransplantation of bone marrow mononuclear cells and umbilical cord mesenchymal stem 
cells in avascular necrosis of the femoral head. Transplant Proc. 2014;46(1): 151-5 Epub 2014/02/11.

36. Chen C, Qu Z, Yin X, Shang C, Ao Q, Gu Y, et al. Efficacy of umbilical cordderived mesenchymal stem cell-based therapy for osteonecrosis of the femoral head: A three-year follow-up study. Mol Med Rep. 2016;14(5):420915 Epub 2016/10/26.

37. Muschler GF, Boehm C, Easley K. Aspiration to obtain osteoblast progenitor cells from human bone marrow: the influence of aspiration volume. J Bone Joint Surg Am. 1997;79(11):1699-709 Epub 1997/12/31.

38. Hernigou P, Poignard A, Beaujean F, Rouard H. Percutaneous autologous bone-marrow grafting for nonunions. Influence of the number and concentration of progenitor cells. J Bone Joint Surg Am. 2005;87(7):1430-7 Epub 2005/07/05.

39. Hernigou P, Trousselier M, Roubineau F, Bouthors C, Chevallier N, Rouard H, et al. Stem Cell Therapy for the Treatment of Hip Osteonecrosis: A 30-Year Review of Progress. Clin Orthop Surg. 2016;8(1):1-8 Epub 2016/03/02.

40. Hernigou P, Poignard A, Zilber S, Rouard H. Cell therapy of hip osteonecrosis with autologous bone marrow grafting. Indian J Orthop. 2009:43(1):40-5 Epub 2009/09/16

41. Aarvold A, Smith JO, Tayton ER, Jones AM, Dawson Jl, Lanham S, et al. From bench to clinic and back: skeletal stem cells and impaction bone grafting for regeneration of bone defects. J Tissue Eng Regen Med. 2014;8(10):77986 Epub 2012/10/06.

42. Aarvold A, Smith JO, Tayton ER, Jones AM, Dawson JI, Lanham S, et al. A tissue engineering strategy for the treatment of avascular necrosis of the femoral head. Surgeon. 2013;11(6):319-25 Epub 2013/04/02.

43. Kang JS, Moon KH, Kim BS, Kwon DG, Shin SH, Shin BK, et al. Clinical results of auto-iliac cancellous bone grafts combined with implantation of autologous bone marrow cells for osteonecrosis of the femoral head: a minimum 5-year follow-up. Yonsei Med J. 2013;54(2):510-5 Epub 2013/02/01.

44. Aoyama T, Goto K, Kakinoki R, Ikeguchi R, Ueda M, Kasai Y, et al. An exploratory clinical trial for idiopathic osteonecrosis of femoral head by cultured autologous multipotent mesenchymal stromal cells augmented with vascularized bone grafts. Tissue Eng B Rev. 2014;20(4):233-42 Epub 2014/03/07.

45. Zhao D, Liu B, Wang B, Yang L, Xie H, Huang S, et al. Autologous bone marrow mesenchymal stem cells associated with tantalum rod implantation and vascularized iliac grafting for the treatment of end-stage osteonecrosis of the femoral head. Biomed Res Int. 2015;2015:240506 Epub 2015/03/25.

46. Yamasaki T, Yasunaga Y, Ishikawa M, Hamaki T, Ochi M. Bone-marrowderived mononuclear cells with a porous hydroxyapatite scaffold for the treatment of osteonecrosis of the femoral head: a preliminary study. J Bone Joint Surg Br. 2010;92(3):337-41 Epub 2010/03/02.

47. Liu Y, Liu S, Su X. Core decompression and implantation of bone marrow mononuclear cells with porous hydroxylapatite composite filler for the treatment of osteonecrosis of the femoral head. Arch Orthop Trauma Surg. 2013;133(1):125-33 Epub 2012/10/17.

48. Pak J. Autologous adipose tissue-derived stem cells induce persistent bonelike tissue in osteonecrotic femoral heads. Pain physician. 2012;15(1):75-85 Epub 2012/01/25.

49. Pilge H, Bittersohl B, Schneppendahl J, Hesper T, Zilkens C, Ruppert M, et al. Bone Marrow Aspirate Concentrate in Combination With Intravenous Iloprost Increases Bone Healing in Patients With Avascular Necrosis of the Femoral Head: A Matched Pair Analysis. Orthop Rev. 2016;8(4):6902 Epub 2017/05/17.

50. Mishima H, Sugaya H, Yoshioka T, Aoto K, Wada H, Akaogi H, et al. The safety and efficacy of combined autologous concentrated bone marrow grafting and low-intensity pulsed ultrasound in the treatment of osteonecrosis of the femoral head. Eur J Orthop Surg Traumatol. 2016;26(3):293-8.

51. Wang Y, Han ZB, Song YP, Han ZC. Safety of mesenchymal stem cells for clinical application. Stem Cells Int. 2012;2012:652034 Epub 2012/06/12

52. Brederlau A, Correia AS, Anisimov SV, Elmi M, Paul G, Roybon L, et al. Transplantation of human embryonic stem cell-derived cells to a rat model of Parkinson's disease: effect of in vitro differentiation on graft survival and teratoma formation. Stem Cells. 2006;24(6):1433-40 Epub 2006/03/25.

53. Seamon J, Keller T, Saleh J, Cui Q. The pathogenesis of nontraumatic osteonecrosis. Arthritis. 2012;2012:601763 Epub 2012/12/18.

54. Balsam LB, Wagers AJ, Christensen JL, Kofidis T, Weissman IL, Robbins RC. Haematopoietic stem cells adopt mature haematopoietic fates in ischaemic myocardium. Nature. 2004;428(6983):668-73 Epub 2004/03/23.

55. Yan Z, Hang D, Guo C, Chen Z. Fate of mesenchymal stem cells transplanted to osteonecrosis of femoral head. J Orthop Res. 2009:27(4): 442-6 Epub 2008/10/18.
56. Jin H, Xu T, Chen Q, Wu C, Wang P, Mao Q, et al. The Fate and Distribution of Autologous Bone Marrow Mesenchymal Stem Cells with Intra-Arterial Infusion in Osteonecrosis of the Femoral Head in Dogs. Stem Cells Int. 2016; 2016:8616143 Epub 2016/01/19.

57. Ciapetti G, Granchi D, Fotia C, Savarino L, Dallari D, Del Piccolo N, et al. Effects of hypoxia on osteogenic differentiation of mesenchymal stromal cells used as a cell therapy for avascular necrosis of the femoral head. Cytotherapy. 2016;18(9):1087-99 Epub 2016/07/17.

58. Fan L, Liu R, Li J, Shi Z, Dang X, Wang K. Low oxygen tension enhances osteogenic potential of bone marrow-derived mesenchymal stem cells with osteonecrosis-related functional impairment. Stem Cells Int. 2015;2015: 950312 Epub 2015/02/19.

59. Fan L, Zhang C, Yu Z, Shi Z, Dang X, Wang K. Transplantation of hypoxia preconditioned bone marrow mesenchymal stem cells enhances angiogenesis and osteogenesis in rabbit femoral head osteonecrosis. Bone. 2015;81:544-53 Epub 2015/09/13.

60. Hung SP, Ho JH, Shih YR, Lo T, Lee OK. Hypoxia promotes proliferation and osteogenic differentiation potentials of human mesenchymal stem cells. J Orthop Res. 2012;30(2):260-6 Epub 2011/08/03.

61. Ding H, Chen S, Yin JH, Xie XT, Zhu ZH, Gao YS, et al. Continuous hypoxia regulates the osteogenic potential of mesenchymal stem cells in a timedependent manner. Mol Med Rep. 2014;10(4):2184-90 Epub 2014/08/12.

62. Fotia C, Massa A, Boriani F, Baldini N, Granchi D. Prolonged exposure to hypoxic milieu improves the osteogenic potential of adipose derived stem cells. J Cell Biochem. 2015;116(7):1442-53 Epub 2015/02/05.

63. Dominici M, Le Blanc K, Mueller I, Slaper-Cortenbach I, Marini F, Krause D, et al. Minimal criteria for defining multipotent mesenchymal stromal cells. The International Society for Cellular Therapy position statement. Cytotherapy. 2006;8(4):315-7 Epub 2006/08/23.

64. Perez-Silos V, Camacho-Morales A, Fuentes-Mera L. Mesenchymal Stem Cells Subpopulations: Application for Orthopedic Regenerative Medicine. Stem Cells Int. 2016;2016:3187491 Epub 2016/10/12.

65. Levi B, Wan DC, Glotzbach JP, Hyun J, Januszyk M, Montoro D, et al. CD105 protein depletion enhances human adipose-derived stromal cell osteogenesis through reduction of transforming growth factor beta1 (TGFbeta1) signaling. J Biol Chem. 2011;286(45):39497-509 Epub 2011/09/29.

66. Leyva-Leyva M, Barrera L, Lopez-Camarillo C, Arriaga-Pizano L, OrozcoHoyuela G, Carrillo-Casas EM, et al. Characterization of mesenchymal stem cell subpopulations from human amniotic membrane with dissimilar osteoblastic potential. Stem Cells Dev. 2013;22(8):1275-87 Epub 2012/12/06.

67. Leyva-Leyva M, Lopez-Diaz A, Barrera L, Camacho-Morales A, HernandezAguilar F, Carrillo-Casas EM, et al. Differential Expression of Adhesion-Related Proteins and MAPK Pathways Lead to Suitable Osteoblast Differentiation of Human Mesenchymal Stem Cells Subpopulations. Stem Cells Dev. 2015; 24(21):2577-90 Epub 2015/08/01.

68. Aslan H, Zilberman Y, Kandel L, Liebergall M, Oskouian RJ, Gazit D, et al. Osteogenic differentiation of noncultured immunoisolated bone marrowderived CD105+ cells. Stem Cells. 2006;24(7):1728-37 Epub 2006/04/08.

69. Jarocha D, Lukasiewicz E, Majka M. Adventage of mesenchymal stem cells (MSC) expansion directly from purified bone marrow CD105+ and CD271+ cells. Folia Histochem Cytobiol. 2008:46(3):307-14 Epub 2008/12/06.

70. Yamamoto M, Nakata H, Hao J, Chou J, Kasugai S, Kuroda S. Osteogenic Potential of Mouse Adipose-Derived Stem Cells Sorted for CD90 and CD105 In Vitro. Stem Cells Int. 2014;2014:576358 Epub 2014/10/11.

71. Rada T, Reis RL, Gomes ME. Distinct stem cells subpopulations isolated from human adipose tissue exhibit different chondrogenic and osteogenic differentiation potential. Stem Cell Rev. 2011;7(1):64-76 Epub 2010/04/17.

72. Itoh H, Nishikawa S, Haraguchi T, Arikawa Y, Eto S, Hiyama M, et al. Aldehyde dehydrogenase activity helps identify a subpopulation of murine adipose-derived stem cells with enhanced adipogenic and osteogenic differentiation potential. World J Stem Cells. 2017;9 (10):179-86 Epub 2017/11/07.

73. Tang TT, Lu B, Yue B, Xie XH, Xie YZ, Dai KR, et al. Treatment of osteonecrosis of the femoral head with hBMP-2-gene-modified tissue-engineered bone in goats. J Bone Joint Sur Br. 2007:(1):127-9 Epub 2007/01/30.

74. Peng WX, Wang L. Adenovirus-Mediated Expression of BMP-2 and BFGF in Bone Marrow Mesenchymal Stem Cells Combined with Demineralized Bone Matrix For Repair of Femoral Head Osteonecrosis in Beagle Dogs. Cell Physiol Biochem. 2017;43(4):1648-62 Epub 2017/10/19.

75. Ma XW, Cui DP, Zhao DW. Vascular endothelial growth factor/bone morphogenetic protein-2 bone marrow combined modification of the 
mesenchymal stem cells to repair the avascular necrosis of the femoral head. Int J Clin Exp Med. 2015;8(9):15528-34 Epub 2015/12/03.

76. Hang D, Wang Q, Guo C, Chen Z, Yan Z. Treatment of osteonecrosis of the femoral head with VEGF165 transgenic bone marrow mesenchymal stem cells in mongrel dogs. Cells Tissues Organs. 2012;195(6):495-506 Epub 2011/11/08.

77. Wen Q, Ma L, Chen YP, Yang L, Luo W, Wang XN. Treatment of avascular necrosis of the femoral head by hepatocyte growth factor-transgenic bone marrow stromal stem cells. Gene Ther. 2008;15(23):1523-35 Epub 2008/07/18

78. Pan ZM, Zhang Y, Cheng XG, Gao GC, Wang XR, Cao K. Treatment of Femoral Head Necrosis With Bone Marrow Mesenchymal Stem Cells Expressing Inducible Hepatocyte Growth Factor. Am J Ther. 2016;23(6): e1602-e11 Epub 2015/07/15

79. Ratajczak MZ, Jadczyk T, Pedziwiatr D, Wojakowski W. New advances in stem cell research: practical implications for regenerative medicine. Pol Arch Med Wewn. 2014:124(7-8):417-26 Epub 2014/06/24.

80. Konala VB, Mamidi MK, Bhonde R, Das AK, Pochampally R, Pal R. The current landscape of the mesenchymal stromal cell secretome: A new paradigm for cell-free regeneration. Cytotherapy. 2016;18(1):13-24 Epub 2015/12/04.

81. Phinney DG, Pittenger MF. Concise Review: MSC-Derived Exosomes for CellFree Therapy. Stem Cells. 2017;35(4):851-8 Epub 2017/03/16.

82. Colombo M, Raposo G, Thery C. Biogenesis, secretion, and intercellular interactions of exosomes and other extracellular vesicles. Annu Rev Cell Dev Biol. 2014;30:255-89 Epub 2014/10/08

83. Tkach M, Thery C. Communication by Extracellular Vesicles: Where We Are and Where We Need to Go. Cell. 2016;164(6):1226-32 Epub 2016/03/12.

84. Guo SC, Tao SC, Yin WJ, Qi X, Sheng JG, Zhang CQ. Exosomes from Human Synovial-Derived Mesenchymal Stem Cells Prevent Glucocorticoid-Induced Osteonecrosis of the Femoral Head in the Rat. Int J Biol Sci. 2016;12(10): 1262-72 Epub 2016/10/22.

85. Liu X, Li Q, Niu X, Hu B, Chen S, Song W, et al. Exosomes Secreted from Human-Induced Pluripotent Stem Cell-Derived Mesenchymal Stem Cells Prevent Osteonecrosis of the Femoral Head by Promoting Angiogenesis. Int J Biol Sci. 2017:13(2):232-44 Epub 2017/03/04

86. Murphy MB, Moncivais K, Caplan Al. Mesenchymal stem cells: environmentally responsive therapeutics for regenerative medicine. Exp Mo Med. 2013;45:e54 Epub 2013/11/16.

87. Salido-Guadarrama I, Romero-Cordoba S, Peralta-Zaragoza O, HidalgoMiranda A, Rodriguez-Dorantes M. MicroRNAs transported by exosomes in body fluids as mediators of intercellular communication in cancer. OncoTargets Ther. 2014;7:1327-38 Epub 2014/08/06.

88. Bellingham SA, Guo BB, Coleman BM, Hill AF. Exosomes: vehicles for the transfer of toxic proteins associated with neurodegenerative diseases? Front Physiol. 2012;3:124 Epub 2012/05/09. 